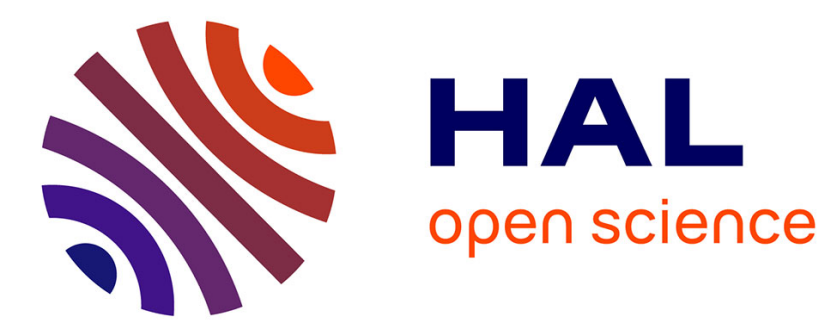

\title{
L'imagerie cérébrale à l'épreuve de la psychanalyse Rémy Potier
}

\section{To cite this version:}

Rémy Potier. L'imagerie cérébrale à l'épreuve de la psychanalyse. Recherches en psychanalyse, 2009, Psychanalyse, psychopathologie cognitive et neurosciences: quel débat?, 1 (7), pp.79-86. 10.3917/rep.007.0079 . hal-01516341

\section{HAL Id: hal-01516341 https://hal.science/hal-01516341}

Submitted on 12 Jul 2017

HAL is a multi-disciplinary open access archive for the deposit and dissemination of scientific research documents, whether they are published or not. The documents may come from teaching and research institutions in France or abroad, or from public or private research centers.
L'archive ouverte pluridisciplinaire HAL, est destinée au dépôt et à la diffusion de documents scientifiques de niveau recherche, publiés ou non, émanant des établissements d'enseignement et de recherche français ou étrangers, des laboratoires publics ou privés. 


\title{
Recherches en Psychanalyse - Research in Psychoanalysis
}

7 | 2009 - Psychanalyse, psychopathologie cognitive et neurosciences : quel débat ?

Psychoanalysis, Cognitive Psychopathology, and the Neurosciences: What is the Debate?

\section{L'imagerie cérébrale à l'épreuve de la psychanalyse}

\author{
Brain imaging under the test of psychoanalysis
}

Rémy Potier

\section{Résumé :}

L'article propose d'interroger, à partir de la psychanalyse, quelques interactions possibles avec les neurosciences. Il s'agit de spécifier la particularité de l'investigation psychanalytique de la technique, notamment de l'imagerie cérébrale, pour en questionner les présupposés. Ce que révèle la prise en compte de toutes les dimension du dispositif, c'est qu'une méthodologie interdisciplinaire permet de circonscrire les limites de ce qui est observé. L'hypothèse du "nouvel inconscient " telle que la développe Lionel Naccache est discutée à cette occasion et mise à l'épreuve de ce qu'enseigne la clinique. L'argumentation de cet article propose l'idée que le dialogue entre psychanalyse et neurosciences apparaît particulièrement fécond si la délimitation des champs et des méthodes se cherche et se précise, sans ambiguïté.

\section{Abstract :}

This article examines some possible interactions between the neurosciences and psychoanalysis, in order to specify the particularities of psychoanalytic investigation of technology, specifically regarding brain imaging, and question its basic assumptions. Taking into account all the aspects of this medical procedure, we find that interdisciplinary approach enables us to define the boundaries of the field of observation. The hypothesis of a "new unconscious", as developed by Lionel Naccache, is also discussed and tested by clinical experience. The author argues that a dialogue between psychoanalysis and the neurosciences appears especially fruitful if we seek to unambiguously delimit and precise its exact field and methods.

Mots-clefs : imagerie médicale, neurosciences, psychanalyse, interdisciplinaire, pulsion scopique Keywords : medical imaging, neurosciences, psychoanalysis, interdisciplinary, drive scopique

\section{Plan :}

L'imagerie fonctionnelle, comme dispositif, induit une nouvelle représentation du corps et de l'image de soi

De la vision au regard, enjeu de l'observatoire comme dispositif

Regards croisés entre neuroscience et psychanalyse : discussion à propos des enjeux épistémologiques 
Les appareils mesurent désormais les différentes réflexions ainsi que les éloignements des différentes parties du corps du sujet pour en reconstituer l'image tridimensionnelle et animée : il s'agit d'une reconstitution. II est en effet possible de voir l'image d'un fœtus, né avant de naître, ou encore tel organe apparaissant par une image sur l'écran. Mais quelle image ? La puissance informatique y est bien ici pour quelque chose. Elle a mis en œuvre les traitements du signal établi par les recherches en mathématiques : la reconstitution d'un volume à partir de ses tranches, opération lourde en calcul. De plus, pour explorer davantage le corps humain, les physiciens ont associé deux inventions des années 1960 : le transistor et le laser, avec les techniques de transmission du signal.

Depuis peu, c'est la totalité du spectre électromagnétique où chaque longueur d'onde détermine les caractéristiques d'un objet qui est maîtrisée. Dernièrement, ce sont la palpation acoustique de l'élasticité d'un organe ainsi que la vision superficielle par rayons $T$ qui ont été rendus possibles. Les biologistes et les chimistes exploitent quant à eux les spécificités fonctionnelles des cellules pour accrocher sur des molécules réceptrices, des émetteurs de lumières ou des molécules radioactives. Grâce à leur connaissance du fonctionnement des cellules, il est permis d'avancer des diagnostics de dysfonctionnement.

Dans certains cas, il est possible de véhiculer des principes actifs jusqu'à une cible pour une activation à l'endroit choisi, comme dans les examens coronariens ayant pour objectif de diagnostiquer les sujets cardiaques. Ainsi, informaticiens, physiciens, chimistes et biologistes sont à l'ouvrage dans ce domaine rejoint par les astrophysiciens, contribuant à l'examen de l'œil par leur technique d'observation qui élime les perturbations.

Une telle armada de compétences scientifiques et techniques peut faire rêver, néanmoins il semble temps de se réveiller pour prendre acte de ce qui échappe aux examens, soit ce que produit cette modélisation de l'intime sur le sujet. Riche de tous les possibles que les techniques liées au virtuel permettent, la réalité augmentée rencontre les difficultés relevées et révélées par les nouvelles technologies dans le champ social.

celle du problème posé par le primat du visuel et celle de l'épistémologie concernant la discussion que la psychanalyse peut tenir avec les neurosciences.

Pour nous concentrer sur le second point, au cœur de la thématique ici travaillée, il convient néanmoins de saisir l'enjeu de la première hypothèse. Pour déployer mon argumentation, je voudrais me laisser guider par ce double questionnement :

Quel est le statut des phénomènes mis à jour dans ce principe d'exhaustivité visuel ?

Quelles en sont enfin les conséquences épistémologiques?

Pour répondre, je m'attarderai sur les trois dimensions que je viens d'évoquer, en mettant en relief trois idées :

L'imagerie fonctionnelle doit être envisagée comme un dispositif, notamment pour saisir la portée du discours produit à travers la méthode qui en résulte.

La vision n'est pas le regard, ce qui a une conséquence épistémologique.

Les phénomènes observés par les neurosciences sont à saisir à partir de leur point de butée, tant pour éviter les confusions d'interprétation, que pour mettre au jour ce que la technique ne peut saisir.

L'imagerie fonctionnelle, comme dispositif, induit une nouvelle représentation du corps et de l'image de soi

L'imagerie fonctionnelle, médiation technique productrice d'images lisibles par le spécialiste, doit être saisie dans son paysage. Son procédé est lié aux développements récents indexés aux productions des technosciences, caractéristiques de notre époque. II peut être tout d'abord 
heuristique d'aborder l'imagerie fonctionnelle à partir de ce que Michel Foucault met à jour à propos du dispositif :

Ce que j'essaie de repérer sous ce nom, [...] un ensemble résolument hétérogène comportant des discours, des institutions, des aménagements architecturaux, des décisions réglementaires, des lois, des mesures administratives, des propositions philosophiques, morales, philanthropiques ; bref, du dit aussi bien que du non-dit, voilà les éléments du dispositif. Le dispositif luimême c'est le réseau qu'on établit entre ces éléments [...] par dispositif, j'entends une sorte - disons - de formation qui, à un moment donné, a eu pour fonction majeure de répondre à une urgence. Le dispositif a donc une fonction stratégique dominante...

J'ai dit que le dispositif était de nature essentiellement stratégique, ce qui suppose qu'il s'agit là d'une certaine manipulation de rapports de force, d'une intervention rationnelle et concertée dans ces rapports de forces, soit pour développer dans telle direction soit pour les bloquer, ou pour les stabiliser, les utiliser. Le dispositif, donc, est toujours inscrit dans un jeu de pouvoir, mais toujours lié aussi à une ou à des bornes de savoir, qui en naissent, mais, tout autant, le conditionnent. C'est ça le dispositif : des stratégies de rapports de force supportant des types de savoir, et supportés par eux. ${ }^{1}$

Foucault se propose d'enquêter sur les modes concrets par lesquels les dispositifs agissent à l'intérieur des relations, dans les mécanismes et les jeux de pouvoir. Suivre les liens qui constituent le réseau de ce dispositif permet d'abord de prendre la mesure du refoulement dont il procède dans le champ social, ce qui consiste ici à se laisser interpeller par le « nondit » qui recouvre ces pratiques.

Giorgio Agamben parlerait volontiers de la question de la stratégie à mettre en place face à ces dispositifs.

Or, si stratégie il doit y avoir, selon moi, à penser l'imagerie fonctionnelle en prenant en compte les agencements qui la constituent, c'est à souligner les effets psychiques produits sur le Sujet, sans céder sur une position critique permettant de situer les enjeux épistémologiques en vigueur.
Ainsi, pour Giorgio Agamben, le projet de Foucault met à jour le lien qui renvoie à un ensemble de praxis, de savoirs, de mesures, d'institutions dont le but est de gérer, de gouverner, de contrôler et d'orienter de façon utile les comportements, les gestes et les pensées de l'homme. II traduit le concept de Foucault comme " tout ce qui $a$, d'une manière ou d'une autre, la capacité de capturer, d'orienter, de déterminer, d'intercepter, de modeler, de contrôler, d'assurer les gestes, les conduites, les opinions et les discours des êtres vivants $"{ }^{2}$ La portée est donc large, et la dimension critique doit alors se justifier précisément quand il faut souligner tout ce que permet l'imagerie

On ne saurait plus aujourd'hui parler de notre corps et de son fonctionnement sans recourir au vocabulaire médical et technique. Le corps est pour nous un ensemble d'organes sièges de processus physiologiques et biochimiques. Ainsi, nous désignons et localisons nos maladies selon une géographie et une terminologie de type médical ou technique, même si celles-ci ne recoupent pas forcément la nomenclature officielle. Le savoir nourrit bien la langue et les représentations mais reste hermétique

Aujourd'hui, l'imagerie fonctionnelle se donne comme objectif de rendre visible des anomalies du corps, avant même que le corps ne livre le moindre signe. L'en deçà de la clinique s'institue comme idéal. Le progrès médical fonde un nouveau réel toujours plus efficace, sans penser que la médecine change l'homme.

Les travaux de Roland Gori montrent que la médicalisation de l'existence amoindrit la capacité d'échange avec l'autre en en faisant une machine réparable et modifiable, déplaçant l'exigence dans une conception qui tire plus l'échange vers la consommation économique que vers la relation humaniste.

Le progrès technoscientique est à démystifier et à replacer dans des projets précis qui sachent entendre les demandes qui leur sont adressées. L'implication des nouvelles technologies tient une place dans la vie des sujets contemporains de façon telle que la représentation du corps 
témoigne d'une virtualisation de plus en plus invasive. L'idéologie peut parfois tenir ferme l'idée d'un gain à cette numérisation du corps dont les artistes se sont saisis, à partir de l'imagerie médicale.

Le malaise dans la culture, est aujourd'hui étroitement lié à ces immersions dans l'image dont l'accès et les pratiques sont de plus en plus répandus.

C'est ainsi que l'art du XXe siècle montre du corps ce que les techniques de visualisation ont permis de voir les unes après les autres, s'insinuant dans la culture, au point de modifier le rapport au corps et à ses images.

Les rayons $X$, les photographies en gros plan, la macrophotographie vont être enrôlés au service de l'art. Les manuels de pose pour la radiographie médicale, les documents photographiques sur les maladies de la peau, de la face et de la bouche, sur les monstruosités et malformations, sont aussi utilisés par les artistes.

Yves Michaux dans son article Les visualisations montre bien cette implication des techniques dans l'art contemporain :

Ces techniques de visualisation, au fur et
à mesure qu'elles deviennent plus
puissantes et indolores, deviennent
paradoxalement aussi plus invasives et
agressives. Elles mettent le corps à nu au
propre et au figuré, y compris dans son
intérieur. Elles le poursuivent jusqu'au plus
intime. Elles traquent, dévoilent, exhibent
ce qui était invisible, caché ou secret. Le
réel est laissé sans voile ni possibilité de
retrait, abandonné à la pulsion scopique.
Ces images du corps qu'on croit d'abord
seulement "nouvelles " transforment en
fait la relation au corps.

Cette transformation du corps est motivée par la recherche d'un corps parfait et idéal. Quelles que soient les finalités, thérapeutiques, esthétiques ou ludiques, ces transformations corporelles poursuivent un corps idéal, un corps de rêve qui, par l'intervention médicale, doit devenir corps réel.

L'imagerie fonctionnelle se doit d'être pensée dans ce contexte.
De la vision au regard, enjeu de l'observatoire comme dispositif

Il faut surtout repérer que la vision s'impose comme le mode privilégié de la créance des techniques d'observation, comme l'imagerie fonctionnelle. L'idéal et la force de persuasion dans les communautés scientifiques, trouvent des alliés dans le développement des technosciences grâce auxquelles se déploie une technologie du regard des plus sophistiquées.

Paul-Laurent Assoun fait remarquer que désormais « c'est le dedans de l'organe qui doit être soumis à cet impératif de visibilité, au point de s'imposer comme "photographie" du symptôme " ${ }^{4}$. II y a donc un mouvement qu'il est utile de repérer pour constater et prendre acte de ce que ce regard n'embrasse pas dans sa poussée propre.

Je voudrais montrer sans attendre que l'imagerie fonctionnelle entérine une représentation du corps morcelé, et que chaque morceau de corps a son mode privilégié d'investigation sur le plan technique. Aussi l'histoire de l'imagerie va réaliser le passage du film radiographique à la virtualisation du corps numérisé. Ce passage engage des effets essentiels aux conséquences à la fois psychiques et épistémologiques.

Il faut bien comprendre que s'accomplit le regard clinique dans toute son ampleur, ce que Foucault annonçait en écrivant que "le regard s'accomplira dans sa vérité propre et aura accès à la vérité des choses, $s^{\prime} i l$ se pose en silence sur elles; si tout se tait autour de ce qu'il voit ".

Face au dispositif que propose l'imagerie, c'est la question du regard posé sur l'icône qui doit être décryptée. Penser ce qu'impliquent les techniques d'observation telle que l'imagerie fonctionnelle, c'est reconnaître que son impératif n'est que scopique.

Le regard comme tel n'est pas interrogé comme geste, dans la mesure même où il se trouve pleinement impliqué dans le mode d'observation propre à la science. 
C'est là que la psychanalyse peut s'avérer épistémologiquement très opératoire, grâce à la posture même de Freud et de ce qu'il propose comme ligne méthodologique permettant de mettre à jour ce qui se joue au cœur même du regard.

Or, une telle posture ne se déduit que de la clinique, elle est même, pourrait-on dire, convoquée par elle. Aussi, chez Freud le visuel témoigne-t-il de l'articulation entre le sexuel et le sensoriel, ce qui requiert pour être compris de lever le refoulement, que le regard posé sur l'image, fusse-t-elle scientifique, induit de façon différente chez le béotien et l'initié, seul à médiatiser son regard à partir de son savoir.

Pour mieux en saisir la portée, il s'avère nécessaire de rappeler le champ qu'a ouvert la psychanalyse, depuis Freud, concernant cette épineuse question, afin de penser ce qui se joue immanquablement dans le dispositif imagier. Le dispositif d'imagerie médicale, en engageant pleinement le regard jusque dans son processus technique, s'allie les procédés de l'optique pour permettre à l'objet de la science et du médecin de trouver son mode d'exploration optimum.

Le pas de côté qu'opère la psychanalyse dans la science produit une avancée conséquente pour la compréhension de l'implication de l'inconscient dans la maladie, certes, mais aussi dans tout processus de sublimation et de constitution d'un savoir.

L'impression optique, nous dit Freud dans les Trois essais, reste le chemin par lequel l'excitation libidinale est le plus fréquemment éveillée.

Il convient donc d'avancer dans l'élucidation des spécificités du regard, et ainsi tenir bon la question du plaisir lié à la vision, seule façon de rendre compte de façon précise du Réel, qui ne manque pas de se manifester à cette occasion.

Que se passe-t-il au niveau de l'imagerie fonctionnelle ? Que vient-il se mêler à cette présentation de l'invisible du corps ? De quel désir procède le regard qui en scrute l'image? Quid de celui qu'elle percute, fascine ou même indiffère?
Le pas que propose Lacan inscrit le regard au cœur de l'élucidation :

Dans notre rapport aux choses, tel qu'il est constitué par la voie de la vision, et ordonné dans les figures de la représentation, quelque chose glisse, passe, se transmet, d'étage en étage, pour y être toujours à quelque degré éludé - c'est ça qui s'appelle le regard. ${ }^{5}$

Ce regard, il s'agit de ne pas le perdre de vue. Lacan en propose une écriture, comme contenant en lui-même l'objet $a$ de l'algèbre lacanienne où le sujet vient à choir. C'est ce qui spécifie le champ scopique et engendre la satisfaction qui lui est propre, c'est que là, la chute du sujet reste toujours inaperçue, car " elle se réduit à zéro " nous dit Lacan. Dans la mesure où le regard, en tant qu'objet $a$, peut venir à symboliser le manque central exprimé dans le phénomène de la castration, il laisse le sujet dans l'ignorance de ce qu'il y a au-delà de l'apparence.

C'est aussi à partir de ces considérations que je propose d'interroger la rencontre avec l'image technoscientifique du corps, rencontre dont l'apparence vient masquer ce qui gît dans le regard posé sur l'image.

L'expérience induite par l'imagerie peut être pensée à profit à partir de ce que Lacan propose autour du stade du miroir et qui permet de dégager l'enjeu spéculaire qui en constitue l'épreuve centrale. Lacan y précise que :

[le] processus de maturation physiologique [...] permet au sujet [...] d'intégrer effectivement ses fonctions motrices, et d'accéder à une maîtrise réelle de son corps. [...] La seule vue de la forme totale du corps humain donne au sujet une maîtrise imaginaire de son corps, prématurée par rapport à la maîtrise réelle. [...]

C'est l'aventure originelle par où l'homme fait pour la première fois l'expérience qu'il se voit, se réfléchit et se conçoit autre qu'il n'est - dimension essentielle de l'humain, qui structure toute sa vie fantasmatique.

Et c'est précisément ce qu'il s'agit de repérer dans la clinique pour permettre au sujet d'y retrouver une parole, seule apte à lui restituer son regard dans l'écart nécessaire que le 
dispositif de l'imagerie vient mettre à mal. (préciser par rapport aux neurosciences)

Il en résulte deux conséquences :

- La présentation d'un corps morcelé, représentant de l'intérieur du corps, vient mettre à l'épreuve l'intégrité de l'unité de l'image de soi, précisément par ce qu'elle n'est pas saisissable.

- Le regard comme tel n'est pas assimilable par la technique. L'objet de la technique c'est le visuel, dont le sujet est absent.

Fort de ce point de vue, qu'en résulte-t-il pour le dialogue avec les neurosciences, qui appuient leur théorie sur les observations issues de I'imagerie anatomo-fonctionnelle?

\section{Regards croisés entre neuroscience et} psychanalyse : discussion à propos des enjeux épistémologiques

L'imagerie fonctionnelle connaît une application privilégiée dans l'étude du cerveau, ce que l'on comprend aisément, tant sa méconnaissance était grande, à partir de la simple méthode d'autopsie. Ce que rend possible, de fait, l'imagerie anatomo-fonctionnelle, c'est bien cette possibilité de voir le cerveau vivant, c'està-dire de prendre sur le vif les zones d'activité cérébrale activées à l'occasion de telle ou telle opération. II s'agit donc de comprendre comment fonctionne le cerveau, à partir de la représentation en images, de son activité.

Ces dernières décennies, les neurosciences ont pu tirer profit de nouvelles méthodes d'investigation permettant d'observer le fonctionnement du cerveau au sein de la totalité organique. Le cerveau se révèle communiquer avec le reste de l'organisme par les substances nutritives et les signaux qu'il reçoit et émet. D'emblée, les observations recueillies le sont à partir des paradigmes des théories de l'information et de la communication. Ainsi, constate-t-on que le fonctionnement du cerveau génère des signaux électriques, les potentiels d'action qui se propagent le long des neurones et aboutissent aux organes.
De telles observations sont précieuses et témoignent de ce qui est rendu possible par le biais des techniques d'imagerie. La grande nouveauté, c'est qu'il est désormais possible " de réaliser des investigations du cerveau sans qu'il y ait danger pour le patient ou le volontaire sain et sans ouvrir la boîte crânienne ${ }^{7}{ }^{7}$

Ces techniques d'investigation offrent des informations à la fois morphologiques, en restituant le cerveau d'un particulier dans toutes ses stratifications, et fonctionnelles, c'est-à-dire qu'elles révèlent les fonctions cognitives à partir desquelles s'active le cerveau pour effectuer diverses tâches. Dans son livre L'imagerie cérébrale fonctionnelle, Bernard Mazoyer, propose le modèle selon lequel " l'activité cognitive peut être vue comme un ensemble organisé dans le temps et dans l'espace d'activités neuronales $"{ }^{8}$

Ce qui intéresse précisément les neurosciences c'est de rendre compte des opérations cognitives, saisir le fonctionnement cérébral, ce qui est en soi heuristique, mais nécessite des balises que certains projets semblent ne même pas concevoir.

Que se passe-t-il et quelle est l'utilité de la visibilité du symptôme psychiatrique via l'imagerie cérébrale ? L'imagerie médicale incite les neuropsychiatres à vouloir voir le psychotique qui délire. L'IRM détecte les régions d'activité neuronale en enregistrant les changements des niveaux d'oxygénation sanguine.

Lorsque l'activité neuronale augmente, le débit de sang oxygéné augmente aussi dans une région donnée. Excès dans la quantité de sang oxygéné par rapport au sang non oxygéné. Le signal de résonance magnétique vient signaler l'activité fonctionnelle. C'est donc ce qui se passe dans le corps qui intéresse, sans autre questionnement que celui consistant à prendre en flagrant délit un corps en activité.

Le problème est bien qu'en cherchant à localiser le délire, le regard en vient à voir ce qu'il veut voir dans l'image, à porter son interprétation au-delà de la rencontre et de la logique que s'est frayé le symptôme dans l'histoire du sujet. 
Dans un article du New York Times, Can Brain Scans See Depression, écrit par Benedict Carey le 18 octobre 2005, on peut lire les déceptions des chercheurs en imagerie de la santé mentale, tâtonnant et constatant d'années en années avoir investi des dépenses colossales pour des études en psychiatrie qui se révèleront infécondes.

Si la leçon vient des U.S.A puisse-t-elle inspirer qui s'y réfère. L'air de l'esprit du temps est aux technosciences. Orienter les recherches demandent donc aussi du discernement et de reconnaître les points de butée qui sont corrélatives des conséquences de certaines épistémologies trop scientistes.

Ce que montre Zarifian, c'est que, face à l'image, les biais méthodologiques sont nombreux et rendent difficile l'interprétation de ce que l'on croit mesurer. Or, c'est bien lorsque l'imagerie vise le cerveau qu'elle ouvre aux interprétations les plus discutables. L'image cérébrale serait ainsi le paradigme même du scotome du réel par la méthodologie scientifique. Là où un organe annexe peut être pris sur le vif, en flagrant délit de jouissance, la possibilité d'y déceler une origine organique stricte est certes possible, mais n'épuise pas pour autant la question de la relation qu'entretient le sujet avec sa maladie et donc son corps. Voir où ça jouit n'épuise pas toute question étiologique, mais au contraire finit par vider toute question.

Pour autant, si l'on suit Zarifian, la clinique s'enrichit d'une approche cognitiviste. II s'intéresse aux recherches sur les fonctions du cerveau qui nous mettent en relation avec l'environnement, qui permettent de donner un sens à ce qui se passe, d'élaborer un programme d'action pour agir sur l'environnement en question. Les fonctions cognitives sont les traitements de l'information par le cerveau. Pour lui, réécouter et observer un malade déprimé ou un malade délirant en ayant présent à l'esprit ces différentes étapes du traitement de l'information permet de recueillir des données qui ne sont pas accessibles par l'observation clinique classique. Cette approche est un bel exemple de croisement des modèles sans tentative d'hybridation du savoir.
La scientificité de la psychanalyse a depuis toujours été remise en cause. Freud, pourtant, reprend ses cas et les retravaille. II peut se reprendre, dire qu'il s'est trompé et pose les limites de ce qu'il avance " au moment des connaissances " auquel est parvenue l'investigation psychanalytique. Quand on critique la psychanalyse, on utilise le mot science au singulier, ce qui pose problème. Or, Gaston Bachelard a théorisé la pluralité des raisons, ce qui est l'argument majeur contre toute forme de positivisme, qui ne pose qu'un seul modèle de scientificité.

Une des confusions fondamentales consiste à confondre l'organe et ce qui passe par l'organe. L'inconscient est inobservable en tant que tel, c'est un concept qui permet de mettre en rapport des phénomènes qui sont repérables mais ne sont pas l'inconscient, seulement des effets. Un certain pan des neurosciences n'accepte pas l'idée qu'il y ait de l'inobservable dans les comportements humains.

Les formations de l'inconscient ne seraient pas de l'inconscient pour la conception neuroscientifique. On ne peut pas réduire la question du sens à ce que les neurosciences observent. II y a une part qui échappe aux neurosciences, et ce reste est sans doute ce qu'investit la psychanalyse. Il y a une part du phénomène qui est radicalement inobservable, et réduire l'objet à ce qui est observable est un problème épistémologique.

Selon Kassirer ${ }^{9}$ l'image qu'on lit n'est qu'un modèle de la réalité, aussi les tomographies d'une tumeur ne sont que des représentations de celle-ci ; les représentations en ultrason d'une anomalie donnée sont des ondes sonores converties en images vidéo, et non la propre anomalie. Les technologies par l'image sont marquées par des discussions permanentes sur l'autorité et l'inscription culturelle, visant à déterminer qui aura l'autorité pour définir le rôle et la signification de ces technologies et comment elles seront institutionnalisées.

Le corps et ses images, pris dans des dispositifs dont la nouveauté est tout entière à mesurer, sont largement impensés dans les termes contemporains 


\section{Bibliographie :}

Agamben, G. (2007). Qu'est-ce qu'un dispositif ? Paris : Éd. Payot \& Rivages.

Ansermet, F. O. \& Magistretti, P. (2004). À chacun son cerveau, plasticité neuronale et inconscient. Paris : O. Jacob. Dehaene, S., Naccache, L., Cohen, L., Bihan, D. L., Mangin, J.-F., Poline, J.-B. \& al. (2001). Cerebral mechanisms of word masking and unconscious repetition priming. [Research Support, Non-U.S. Gov't]. Nat Neurosci, 4(7), 752-758.

Foucault, M. (2001). Dits et écrits, 1954-1988. Paris : Gallimard.

Kandel, E. R. (2006). In search of memory: The emergence of a new science of mind. (2006). New York Times Book Review. Kassirer, J. P. (1992). Images in clinical medicine.

[Comment Editorial]. N Engl J Med, 326(12), 829-830.

Kassirer, J. P. (1998). Managing care-should we adopt a new ethic? [Editorial]. N Engl J Med, 339(6), 397-398.

Kassirer, J. P. (2000). Medicine at the turn of the century. [Editorial]. Ann Thorac Surg, 70(2), 351-353.

Lacan, J. (1992). Les Quatre concepts fondamentaux de la psychanalyse. Le Séminaire, XI (1964). Paris : Seuil.

Lacan, J. (1999). Écrits texte intégral (Nouv. éd.). Paris : Seuil.

Mazoyer, B. (2001). L'imagerie cérébrale fonctionnelle. Paris : PUF.

Michaud, Y ; (2005). Visualisations ; In Corbin, A., Courtine, J.-J. \& Vigarello, G. (2006). Histoire du corps. (2006). Paris : Seuil.

Naccache, L. \& Dehaene, S. (2001). The priming method: imaging unconscious repetition priming reveals an abstract representation of number in the parietal lobes. [Research Support, Non-U.S. Gov't]. Cereb Cortex, 11(10), 966-974.

Naccache, L., Blandin, E., \& Dehaene, S. (2002). Unconscious masked priming depends on temporal attention. [Research Support, Non-U.S. Gov't]. Psychol Sci, 13(5), 416-424.
Naccache, L. (2006). Le nouvel inconscient Freud, Christophe Colomb des neurosciences. Paris : O. Jacob. Pautrat, J.-L. (2007). Des puces, des cerveaux et des hommes quand l'électronique dialogue avec le cerveau. Paris : Fayard.

Potier, R. (2007). L'imagerie médicale à l'épreuve du regard : enjeux éthiques de l'expérience face à l'image. Cliniques méditerranéennes : Psychanalyse et Psychopathologie Freudiennes. Vol. 76, 77-90.

Sicard, M. (1991). Le cerveau dans tous ses états. Entretiens avec Michel Desgeorges, Michel Imbert, Alain Prochiantz, Roger Saban, Jean-Pol Tassin, Marie-Hélène Thiébot, Jean-Didier Vincent et Edouard Zarifian. Paris : Presses du CNRS. Zarifian.

\section{Notes :}

${ }^{1}$ Foucault, M. (2001). Dits et écrits, volume II. Paris: Gallimard, p. 299 sq.

${ }^{2}$ Agamben, G. (2007). Qu'est-ce qu'un dispositif ?. Paris : Payot \& Rivages, p. 31.

${ }^{3}$ Michaud, Y. (2005). Visualisations. In Corbin, Alain, Courtine, Jean-Jacques \& Vigarello, Georges (2006). Histoire du corps. (2006). Paris : Seuil, p. 421.

${ }^{4}$ Assoun, P.-L. (2009). L'image médicale à l'épreuve de la psychanalyse. Recherches en psychanalyse, 8|2009.

${ }^{5}$ Lacan, J. (1992). Les Quatre concepts fondamentaux de la psychanalyse. Le Séminaire, XI (1964). Paris : Seuil.

${ }^{6}$ Lacan, J. (1999). Écrits texte intégral (Nouv. éd.). Paris : Seuil.

${ }^{7}$ Pautrat, Jean-Louis (2007). Des puces, des cerveaux et des hommes - Quand l'électronique dialogue avecle cerveau. Paris : Fayard, p. 58.

${ }^{8}$ Mazoyer, Bernard (2001). L'imagerie cérébrale fonctionnelle. Paris : PUF.

${ }^{9}$ Kassirer, J.P. (1992). Images in clinical medicine. The New England Journal of Medicine, vol. 326, $\mathrm{n}^{\circ} 12$, p. 829-839.

\section{L'auteur :}

\section{Rémy Potier}

Docteur en Psychopathologie / Phd. Maître de Conférences en Psychopathologie. Centre de Recherches Psychanalyse, Médecine et Sociétés - EAD N³522. Université Paris VII Diderot Campus Paris Rive Gauche

Bâtiment Olympe de Gouges

11, rue Jean Antoine de Baïf

\section{Référence électronique}

Rémy Potier, " L'imagerie cérébrale à l'épreuve de la psychanalyse ", Recherches en Psychanalyse [En ligne], 07|2009, mis en ligne le $1^{\mathrm{er}}$ juin 2009.

\section{Texte intégral}

\section{Droits d'auteur}

Tous droits réservés

75013 Paris

France 